Vol. 19, $n^{\circ} 2 \mid 2015$

Varia

Alain Denizet, L'affaire Brierre. Un crime insensé à la Belle Époque

préface d'Alain Corbin, Paris, Les Éditions de la Bisquine, 2015, 320 p., ISBN 9791092566086

Jean-Claude Farcy

\title{
OpenEdition
}

Journals

Édition électronique

URL : http://journals.openedition.org/chs/1612

DOI : $10.4000 /$ chs. 1612

ISSN : 1663-4837

Éditeur

Librairie Droz

Édition imprimée

Date de publication : 1 novembre 2015

Pagination : 153-156

ISBN : 978-2-600-01908-8

ISSN : 1422-0857

Référence électronique

Jean-Claude Farcy, «Alain Denizet, L'affaire Brierre. Un crime insensé à la Belle Époque », Crime, Histoire \& Sociétés / Crime, History \& Societies [En ligne], Vol. 19, n² | 2015, mis en ligne le 01 novembre 2017, consulté le 24 septembre 2020. URL : http://journals.openedition.org/chs/1612 ; DOI : https://doi.org/ $10.4000 /$ chs. 1612

Ce document a été généré automatiquement le 24 septembre 2020

(C) Droz 


\section{Alain Denizet, L'affaire Brierre. Un crime insensé à la Belle Époque}

préface d'Alain Corbin, Paris, Les Éditions de la Bisquine, 2015, 320 p., ISBN 9791092566086

Jean-Claude Farcy

\section{RÉFÉRENCE}

Alain Denizet, L'affaire Brierre. Un crime insensé à la Belle Époque, préface d'Alain Corbin, Paris, Les Éditions de la Bisquine, 2015, 320 p., ISBN 9791092566086.

On connaît la vogue, déjà ancienne, des récits d'affaires célèbres. Elle est renouvelée depuis quelques années, pour la France, par une collection départementale des "grandes affaires criminelles» convoquant la plume de journalistes et parfois d'historiens. On aurait grand tort de ranger l'ouvrage d'Alain Denizet dans ce registre des publications destinées au "grand public». Certes le récit est alerte, remarquablement écrit, dans un style à la fois percutant et nuancé, apte à guider le lecteur dans les arcanes d'une affaire criminelle particulièrement originale. Car au-delà du récit précis et méticuleux d'un crime hors du commun, est constamment présente l'analyse d'un historien rigoureux, très au fait de l'histoire de sa région, de la justice et du crime. Impossible de prendre en défaut l'auteur quant à sa documentation - le dossier de procédure riche de plus de 900 pièces, une importante collection de presse nationale et internationale, les sources pénitentiaires coloniales - comme à son exploitation toujours distante et nuancée. Non seulement Alain Denizet brosse, à travers cette affaire, une excellente mise au point sur une enquête judiciaire et un procès criminel au tout début $\mathrm{du} \mathrm{XX}^{\mathrm{e}}$ siècle, mais il donne également matière à réflexion sur une criminalité exceptionnelle, atypique, objet d'une exploitation médiatique particulièrement développée et interférant avec le travail même des gens de justice. 
2 Le crime étudié a lieu dans une petite commune proche de Chartres, à Corancez (Eureet-Loir) : dans la nuit du 21 avril 1901, cinq des enfants de Brierre - un petit cultivateur en même temps entrepreneur de battage - sont assassinés et leur père lui-même est retrouvé le lendemain, apparemment à l'agonie. Mis d'abord sur le compte de vagabonds (les "trimardeux» ou "cheminaux»), le crime est imputé au bout de quelques jours au père qui aurait simulé être victime des assassins. Alain Denizet décrit minutieusement, dans une première partie, la découverte du crime, le traumatisme du village, les premiers pas de l'enquête, ses développements avec la découverte progressive d'indices à charge dissimulés en différents endroits, ainsi que l'extraordinaire emballement médiatique qui s'ensuit. Il faut dire que le forfait commis, dans une région rurale qui conduit à convoquer les références littéraires attendues sur une paysannerie restée un peu « sauvage » (Maupassant, Zola), se situe au sommet de la criminalité - l'enfant a alors détrôné le père dans la hiérarchie des victimes - et semble illustrer parfaitement le climat d'insécurité (la presse dénonce la «crise de la répression»). De plus, ce crime impensable est nié par l'inculpé : Brierre, sans antécédents judiciaires, honnête travailleur, excellent père élevant seul ses enfants depuis le décès de leur mère, nie farouchement en être l'auteur. Au crime qui dépasse l'entendement s'ajoutent l'énigme du mobile et l'incertitude quant à la culpabilité de l'inculpé.

3 En l'absence d'aveu, de témoignages directs, l'instruction est relativement longue pour l'époque - un peu moins de 8 mois - et se fait sous pression médiatique : la scène du crime n'est guère "sanctuarisée ", habitants et journalistes allant et venant dans la cour et les dépendances de la ferme. Le quatrième chapitre décrit avec bonheur la quête d'indices (dont les armes du crime : un coutre de charrue et un couteau), souvent découverts par les journalistes enquêteurs qui suscitent également la libération de la parole des habitants du village ${ }^{1}$. Ceux-ci font bloc, au lendemain du crime, en faveur de Brierre en avançant l'idée d'un crime de " trimardeux » (journaliers étrangers souvent embauchés pour le battage des grains). Puis, quand les charges s'accumulent contre Brierre, unanimement contre lui : on évoque alors sa maîtresse - la fille de ses voisins -, un incendie dont il avait été auparavant victime et dont il pourrait bien avoir été l'auteur, son caractère hautain, voire le fait qu'il ne soit pas né dans la commune, donc un peu "étranger au pays" même s'il y habite depuis longtemps... De belles pages décrivent l'évolution du portrait de Brierre dans l'opinion locale ( «la langue du monde »), de paysan sans histoire, père dévoué à ses enfants, petit cultivateur sérieux à la figure du monstre qu'il est devenu et fustigé par les "procureurs en blouse » du village de Corancez qui tentent d'opérer ainsi la catharsis du crime. La presse s'efforce de combler l'instruction sur son point faible - le mobile - en interrogeant habitants et gardiens de prison pour tenter de percer le «mystère " de cet homme désormais présenté comme dissimulateur, solitaire et taciturne, froid et insensible.

4 Dans le chapitre suivant l'auteur apporte une réflexion intéressante sur ce point, rappelant d'abord les mobiles avancés à l'époque: pour certains, chargé de famille, Brierre aurait supprimé ses enfants pour pouvoir se remarier avec sa maîtresse ; pour d'autres, le geste aurait été accompli pour des raisons pécuniaires, afin d'éviter la faillite. Alain Denizet inscrit ce dernier mobile dans le contexte plus large de la peur du déclassement social. Brierre a pu craindre de perdre, au vu de ses dettes, le statut d'indépendant et de modeste patron qu'il avait acquis par son travail et la force de son caractère, revivant avec douleur son passé (chef de famille à 16 ans à la mort de son 
père), ayant à l'esprit l'exemple désastreux de la faillite de son beau-frère, également entrepreneur de battage, redevenant journalier et perdant l'autorité paternelle. L'auteur suggère l'hypothèse d'une sorte d'épuisement psychique devant son échec perçu comme inévitable et injuste, les blessures qu'il se serait infligées après la mort donnée aux enfants pouvant se lire comme une tentative de suicide. Il avance également, en conclusion, l'hypothèse d'un "déni radical inconscient» (p. 304) qui expliquerait son acharnement, pendant l'instruction, le procès et au bagne, à proclamer son innocence.

5 Ces approches restent prudemment hypothétiques d'autant plus qu'elles ne peuvent s'appuyer sur une expertise psychologique, non réclamée par le juge d'instruction. Le récit du procès (chapitre VI) est familier aux historiens : une cour d'assises à l'étroit dans un tribunal de province envahi par les journalistes, un président très partial soumettant l'accusé à un interrogatoire interminable, des témoins à charge intimidés et contraints de répondre aux répliques de l'accusé se disant victime d'un complot, une défense dépassée prononcée par un avocat parisien déstabilisé par sa radiation prochaine du barreau, des experts n'emportant pas une conviction certaine, et, pour la presse, le moment attendu d'émotion tragique avec le témoignage de soutien à son père apporté par la fille de l'accusée (placée à Paris au moment du crime).

6 La peine capitale prononcée suscite pendant deux mois - fin décembre 1901, début février 1902 - les débats habituels sur l'incompétence des jurés et leur implication locale. Mais la presse - celle d'opinion principalement - met surtout en avant un procès biaisé, les doutes sur une culpabilité non pleinement assurée et agite le spectre de l'erreur judiciaire. Faut-il alors, comme le suggère Alain Denizet, considérer que l'affaire Brierre est à l'origine de deux débats sur la grâce (accordée : la peine capitale est commuée en celle de travaux forcés) et sur la peine de mort (débat parlementaire de février 1902 sur la suppression des crédits au bourreau) ? C'est peut-être un peu grossir l'importance de cette affaire sur ce plan, car beaucoup d'autres seront mobilisées dans le débat de fond devant le Parlement en 1908. Par contre, on prêtera intérêt au chapitre VIII consacré à l'exploitation par les antifreyfusards de l'affaire Brierre, notamment lors des interventions multiples en faveur de la commutation de sa peine capitale. Est ici suggérée une piste intéressante sur l'impact que peut avoir l'affaire Dreyfus sur le traitement médiatique et politique des affaires criminelles. Si les dreyfusards se gardent d'intervenir dans une affaire de droit commun, leurs adversaires dénoncent les mêmes « combines » pour obtenir la grâce, et le même risque d'affaiblissement de l'autorité de l'État, en faisant explicitement le parallèle entre les deux affaires.

7 Dans un dernier chapitre, Alain Denizet suit Brierre de Fresnes au bagne de Guyane où il arrive en janvier 1903. Il sera rapidement affecté comme infirmier à l'hôpital de l'île Royale, tâche qu'il accomplit de manière exemplaire, faisant figure de bagnard modèle aux yeux des autorités pénitentiaires, lesquelles appuient ses démarches constantes pour obtenir la révision de son procès. Jusqu'à sa mort - mars 1910 - il protestera toujours de son innocence.

8 Tels sont, trop rapidement évoqués, le parcours de ce condamné hors norme et quelques-uns des éclairages apportés par l'auteur sur cette affaire. À juste titre, la conclusion de l'ouvrage s'interroge sur l'oubli de cette affaire criminelle depuis le milieu du XXe siècle, alors qu'elle a été l'une des plus médiatisées entre 1870 et 1918, y compris à l'étranger, en rapport naturellement avec l'apogée d'une presse friande de 
crimes à la Une. Plus que le cadre rural, sans doute faut-il remarquer, au-delà d'un crime exceptionnel, que le caractère même de Brierre (un "taiseux ») et surtout les doutes sur l'existence d'une erreur judiciaire ne sont pas faits pour maintenir vivace jusqu'à nos jours le souvenir du crime de Corancez.

9 Alain Denizet nous le fait revivre dans toutes ses dimensions. La force de son travail tient dans une mise en contexte très informée, notamment sur le plan régional et sur la place des jeunes dans le monde rural. En témoignent par exemple les deux chapitres consacrés aux jours précédant le crime et à la biographie d'une " famille ordinaire » : à leur lecture, non seulement on vérifie une fois de plus que les archives d'un procès nous font pénétrer l'intimité des "gens de peu » comme le rappelle Alain Corbin dans sa préface, mais que l'on dispose également des éléments familiaux, sociaux et relationnels qui permettent d'avancer des hypothèses sur cette "énigme» du basculement dans un crime atroce. C'est, nous semble-t-il la voie à suivre pour analyser les crimes "singuliers", exceptionnels, qui fascinent l'opinion et tentent de plus en plus les historiens.

\section{NOTES}

1. L'exploitation médiatique de l'affaire et le rôle des reporters dans l'enquête illustrent parfaitement l'analyse de Dominique Kalifa, L'encre et le sang. Récits de crimes et société à la Belle Époque, Paris, Fayard, 1995, 360 p. 